Original Research

\title{
Comparison of Usability of Touch-screen and Button Cell Phones Among EIderly Users
}

\begin{abstract}
Ali Arabian' ${ }^{1}$ (D), Seyed Abolfazl Zakerian ${ }^{2 *}$
1. MSc Student of Ergonomics, Department of Occupational Health Engineering, School of Public Health, Tehran University of Medical Sciences, Tehran, Iran

2. Associate Professor, Department of Occupational Health Engineering, School of Public Health, Tehran University of Medical Sciences, Tehran, Iran
\end{abstract}

\begin{tabular}{lr}
\hline \multicolumn{2}{c}{ Article Info } \\
\hline Original Article \\
Received: & $2018 / 10 / 01$ \\
Accepted: & $2019 / 02 / 01$ \\
Published Online: & $2019 / 03 / 21$
\end{tabular}

DOI: $10.30699 /$ jergon.7.1.1

Use your device to scan and read the article online

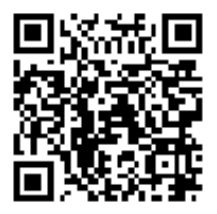

Corresponding Information

Seyed Abolfazl Zakerian, Associate Professor, Department of Occupational Health Engineering, School of Public Health, Tehran University of Medical Sciences, Tehran, Iran

\section{Email:}

zakerian@sina.tums.ac.ir

\section{Abstract}

Background and Objectives: The use of new technologies, particularly mobile phones, is drastically growing on a daily basis. The usability of mobile phones can be determined for different age groups in order to reach a balanced development plan with respect to this technology. The present study aimed to investigate and compare the usability of touch-screen and button cell phones among Iranian elderly users, with respect to objective performance and perceived usability.

Methods: In this cross-sectional study, 20 elders (50\% female) with an average age of were selected by simple random sampling method to measure the usability of touch-screen and button cell phones. In order to assess the objective performance of how elders work with cell phones, tests were conducted including items like typing speed, number of typing errors, taking photo, creating new contacts, and sending text messages. Perceived usability was obtained using a questionnaire of cell phone usability. Furthermore, muscle pressure and mental work load during cell phone use were evaluated by Borg CR10 and NASA-TLX questionnaires, respectively. Data were analyzed conducting statistical analysis tests of T-test and Mann-Whitney by SPSS24 software.

Results: Results revealed that the average typing speed and typing errors in touch-screen cell phones are larger than that in button cell phones. Moreover, the average of perceived usability in touch-screen cell phones (5.8) is higher than that in button cell phones (4.3). Pearson correlation coefficient showed an inverse relationship $(-0.3)$ between perceived usability and mental work load obtained in both types of the cell phones. Findings showed that the maximum perceived muscle pressure when working with a cell phone is felt in the neck area.

Conclusion: According to the obtained results, touch-screen cellphones are suggested for those elders who type more and button cell phones are recommended for those who use the cell phone menu more frequently, namely taking photo, calling, internet, and so forth.

Keywords: Elderly, Cellphone, Usability

Copyright (C) 2019, This is an original open-access article distributed under the terms of the Creative Commons Attribution-noncommercial 4.0 International License which permits copy and redistribute of the material just in noncommercial usages with proper citation.

How to Cite This Article:

Arabian A, Zakerian A. Comparison of Usability of Touch-screen and Button Cell Phones Among Elderly Users. Iran J Ergon. 2019; 7 (1) :1-9 


\title{
E-ISSN: 2345-5365 | مجلة اركونومى | سال V، شمارهُ ا، بهار
}

مقالأ يزوهشى

مقايسٔ كاربرديذيرى گوشى هاى لمسى و دكمهاى در كاربران سالمند

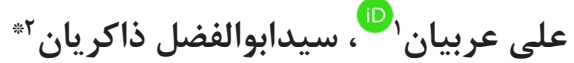

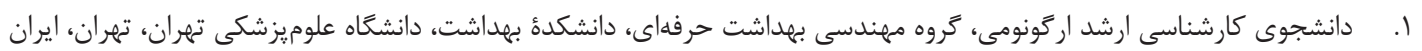

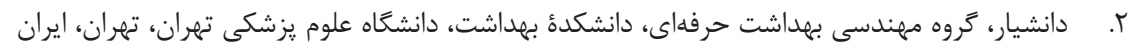

\begin{tabular}{|c|c|}
\hline קكيده & اطلاعات مقاله \\
\hline 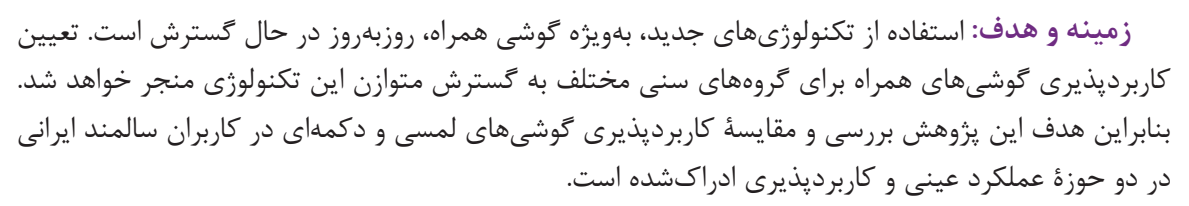 & $\begin{array}{ll}\text { تاريخ وصول: } & \text { تانتشار آنلاين: }\end{array}$ \\
\hline 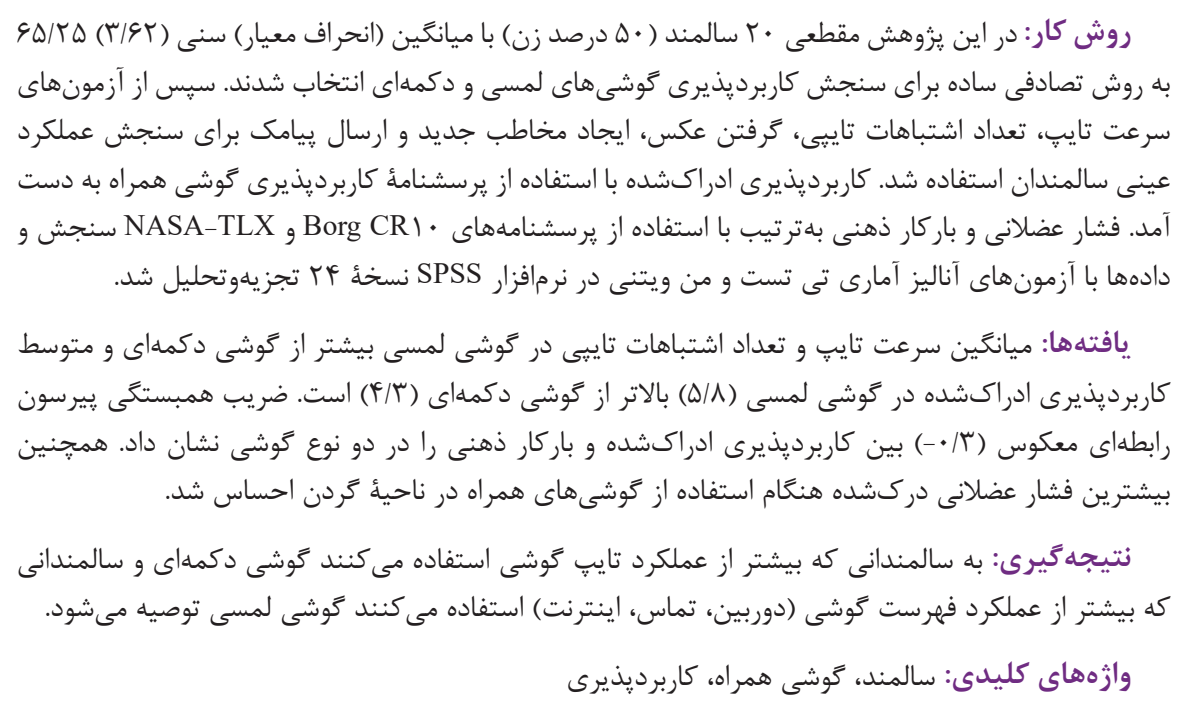 & 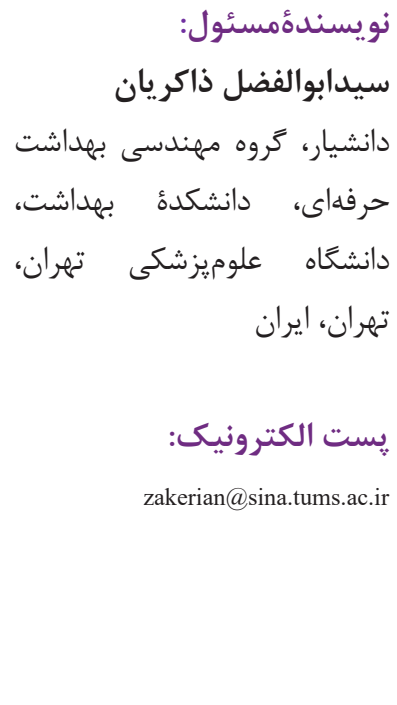 \\
\hline
\end{tabular}

كاربرى سالمندان هستند، زيرا با افزايش سن اختلالات

مقدمه

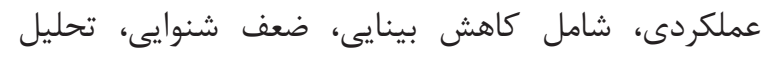

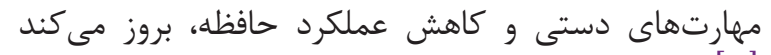

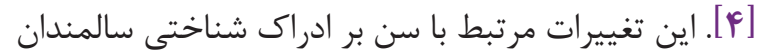

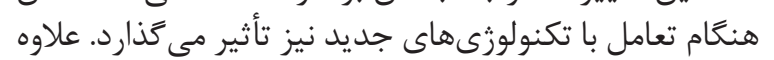

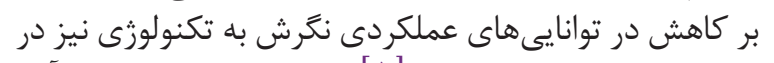

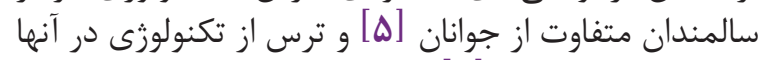
بيشتر از جوانان است [عارت إن.

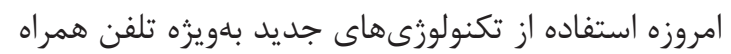

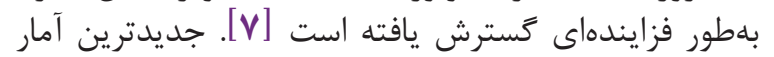

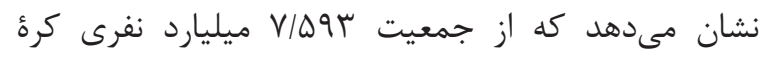

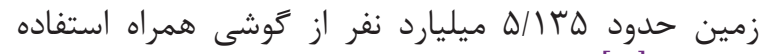

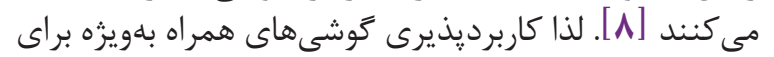

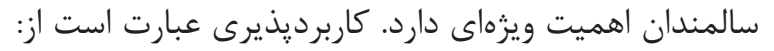

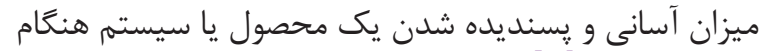

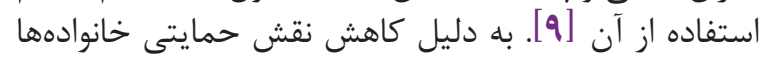

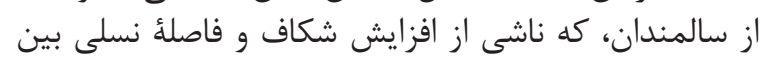

جهان بهسرعت در حال پِير شدن است. تعداد افراد بالاى

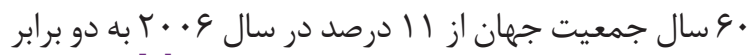

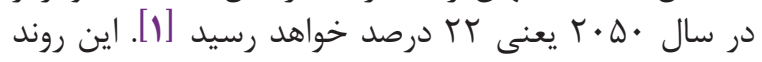

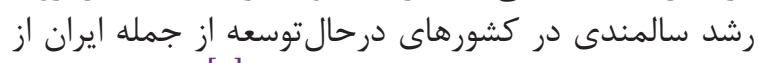

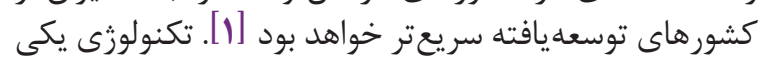

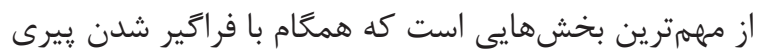

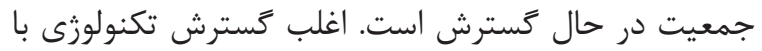

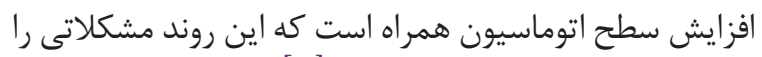

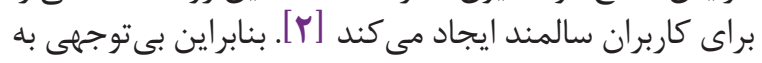

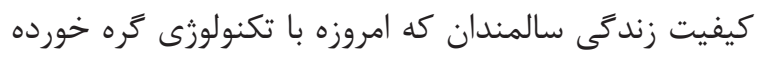

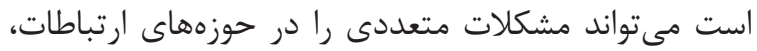
سلامت و استقلال سالمندان ايجاد كند.

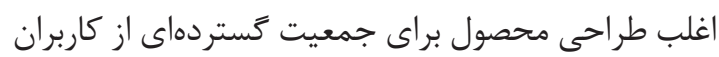

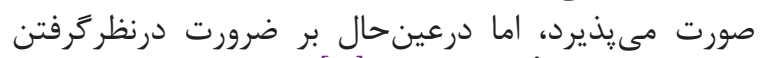

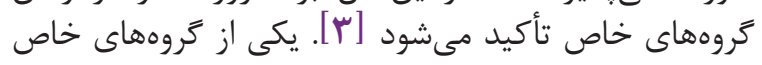


براى اين يزوهش كوشى لمسى سامسونگ مدل Galaxy Prime Ja

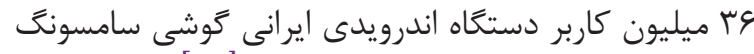

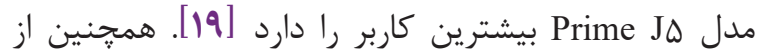

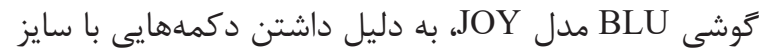

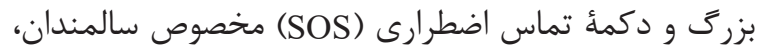

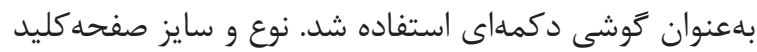

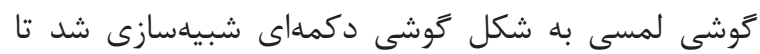

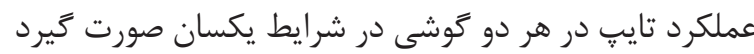

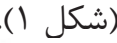
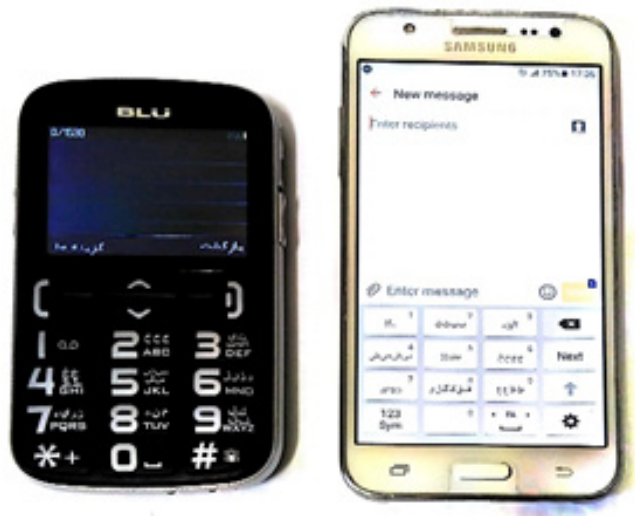

شكل ا. كوشى لمسى مدلGalaxy J5 Prime (شكل سمك سمت راست) و كوشى دكمهاى BLU مدل JOY (شكل سمت جي)

در اين يزوهش دو وظيفه براى شركت كنندكان طراحى

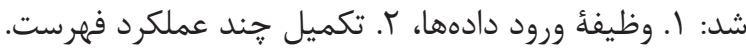

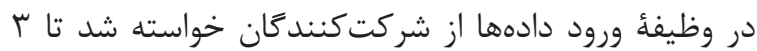

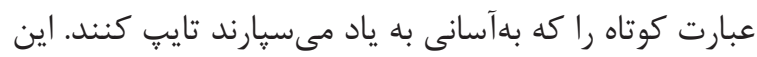

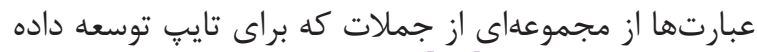

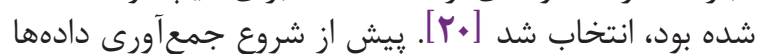

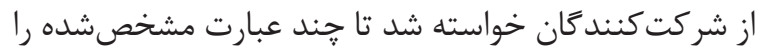

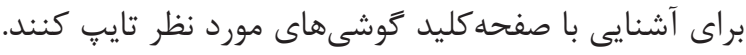

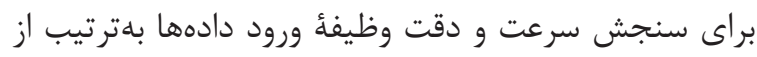

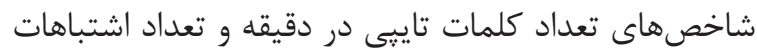

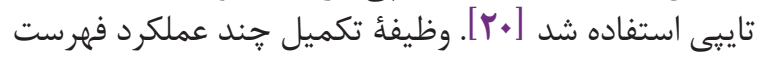

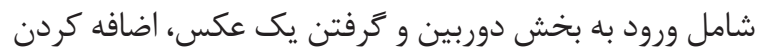

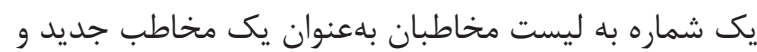

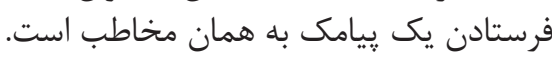

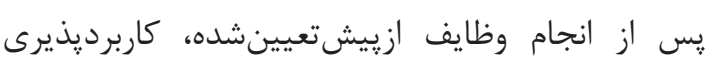

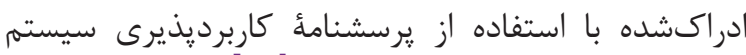

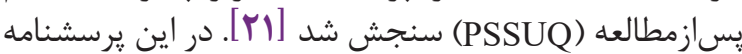

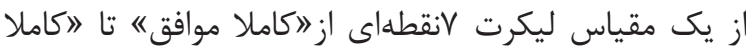

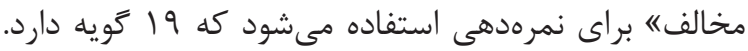

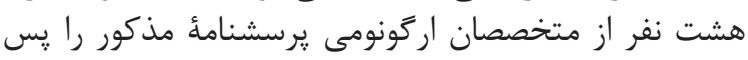

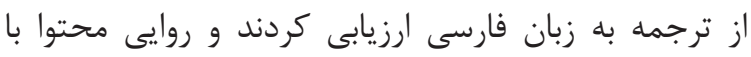

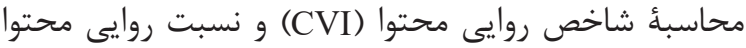

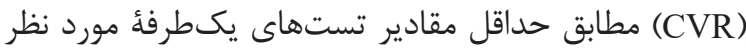
(I9VD) Lawshe

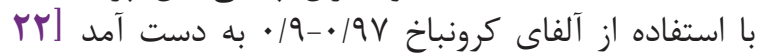

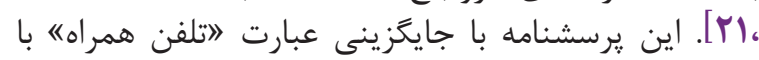

جوانان و سالمندان است، اهميت استفاده از گوشى همراه

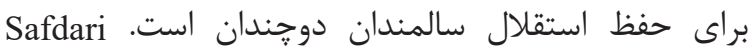

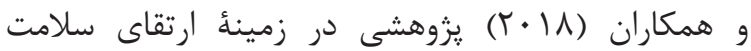

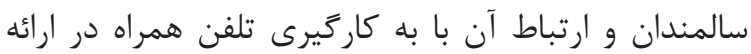

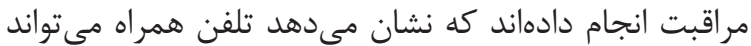

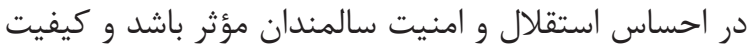
زندگى آنان را ارتقا دهد [ـ1].

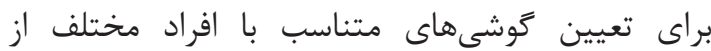

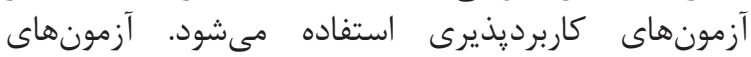

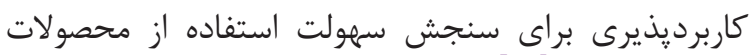

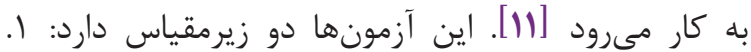

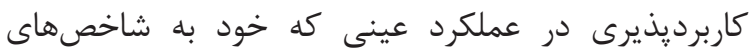

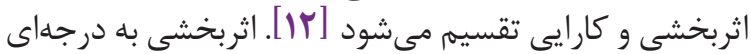

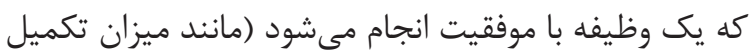

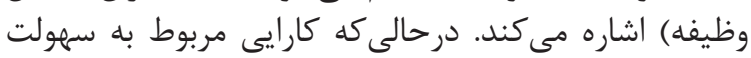
انجام وظيفه است (مانند زمان تكميل وظيفه و و ميزان خان خطار) r. كاربرديذيرى ادراكشده (ذهنى).

كوشىهاى تلفن همراه انواع مختلفى دارد كه بهطور كلى نقي

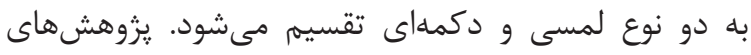

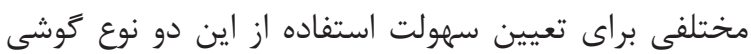

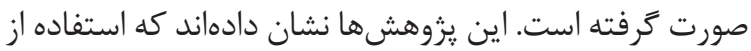

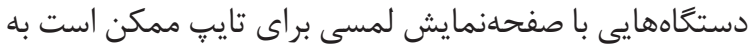

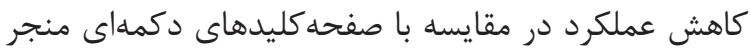

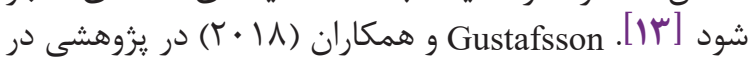

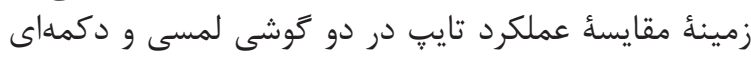

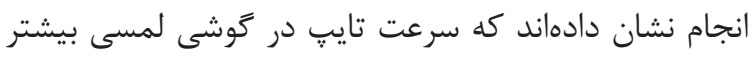

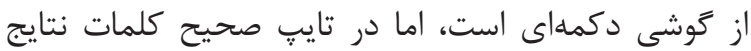

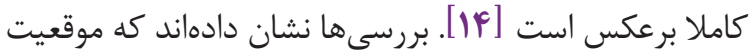

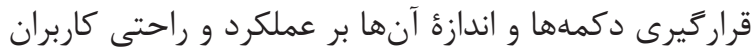

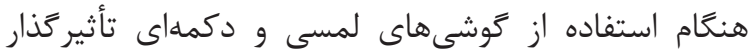

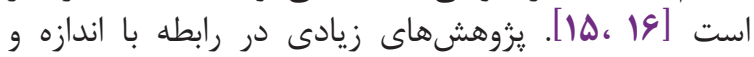

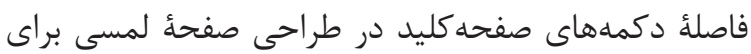

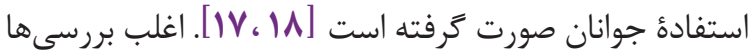

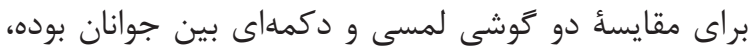

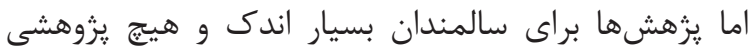

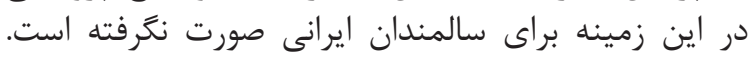

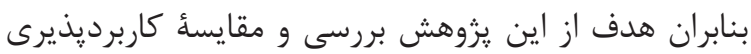

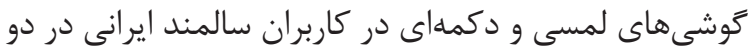
حوزه عملكرد عينى و كاربرديذيرى ادر اكر اكشده است.

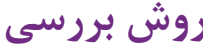

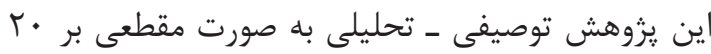

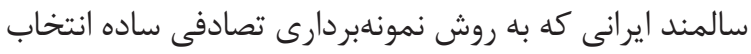

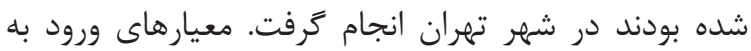

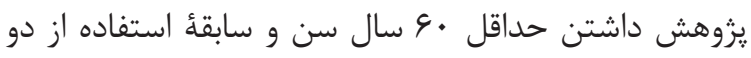

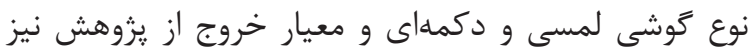

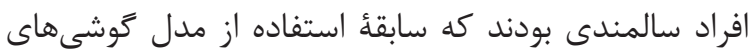

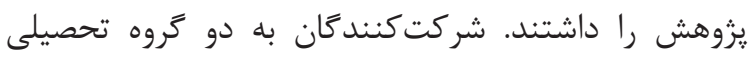

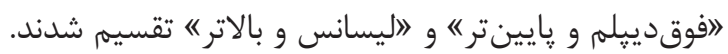




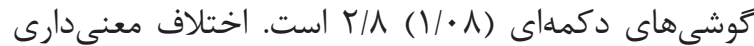

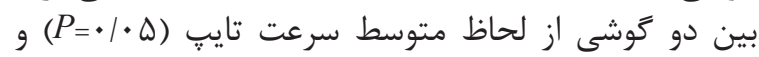

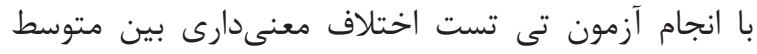

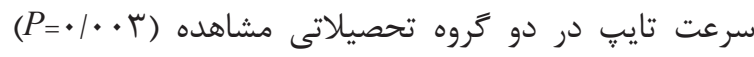

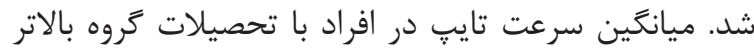

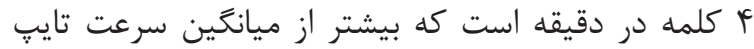

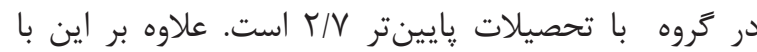

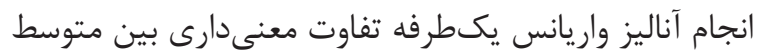

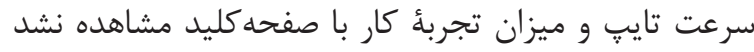
تايتي

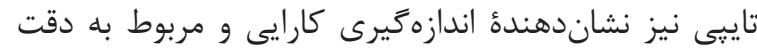

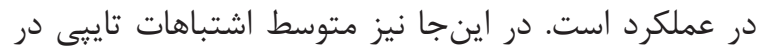

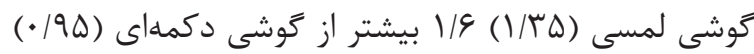

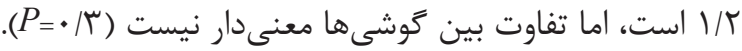

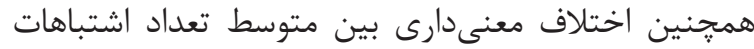

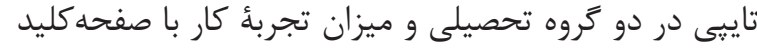

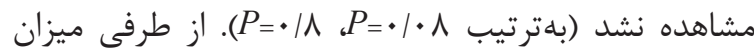

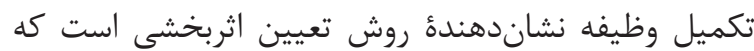

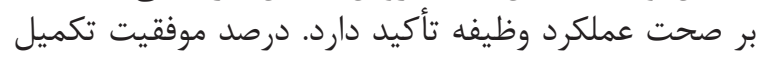

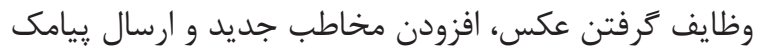

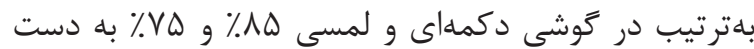

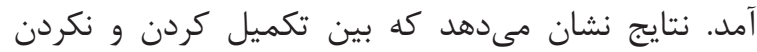

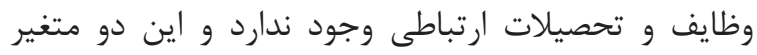

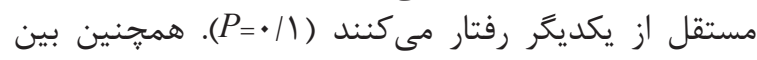

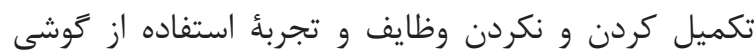

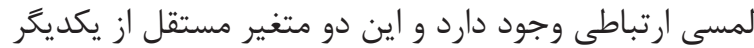
رفتار نمى كنند (

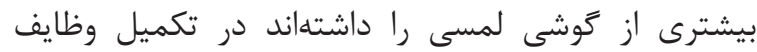

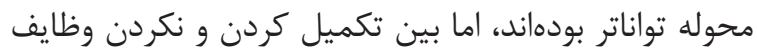

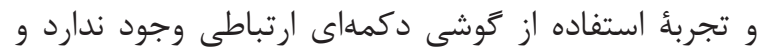

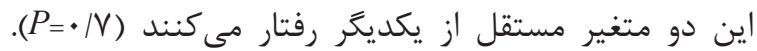

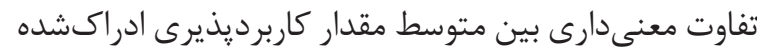

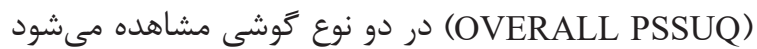

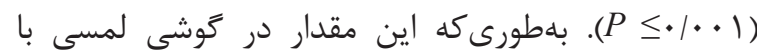

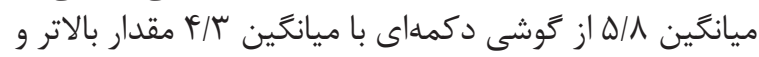
معنى دارى دارد (شكل ؟ ؟).

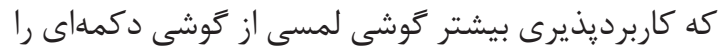

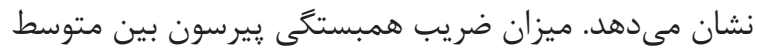

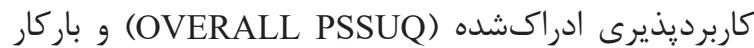

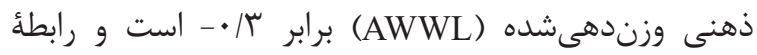

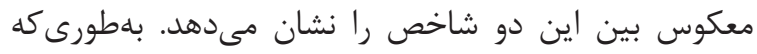

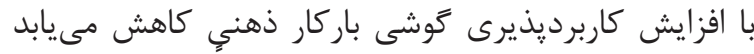

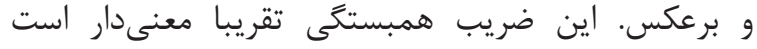
. $(P=\cdot / \cdot 9)$

اطلاعات مربوط به متغيرهاى بار كارى با استفاده از روش دوش

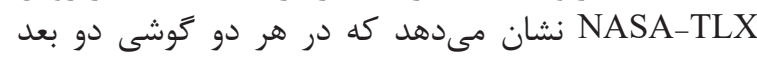

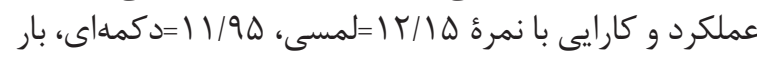

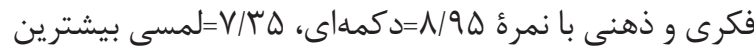

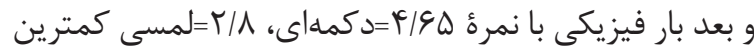

" - (سيستمه اصلاح شد.

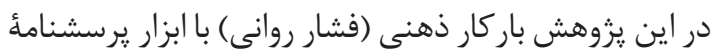

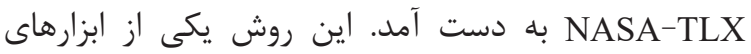

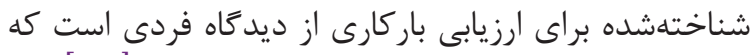

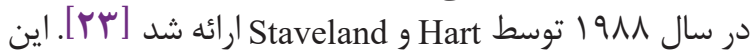

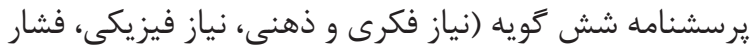

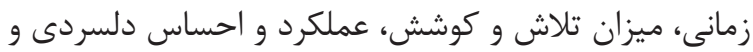

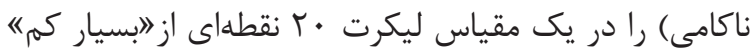

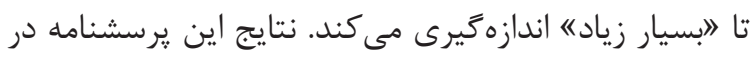

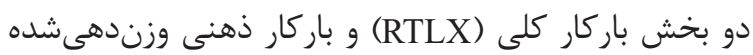

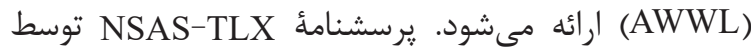
Mohammadi

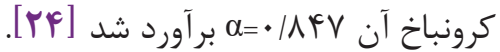
فشار عضلانى دركشده براى كردن، شانه، بازو، ساعد،

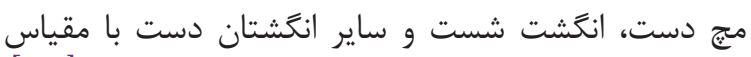

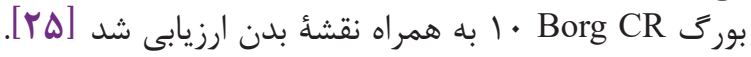

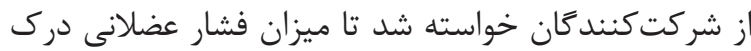

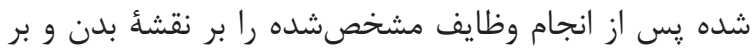

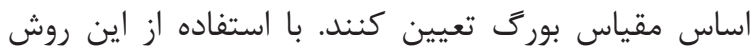

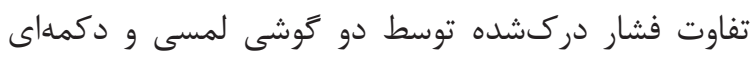

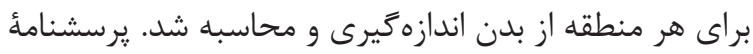

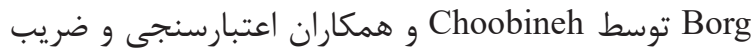

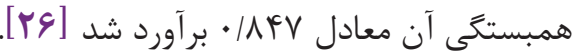

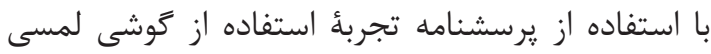

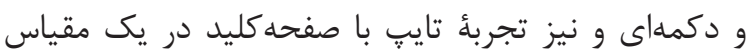

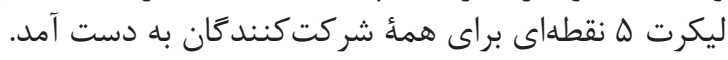

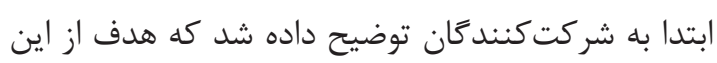

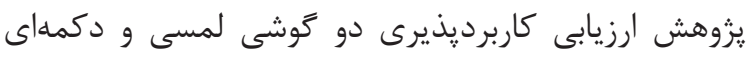

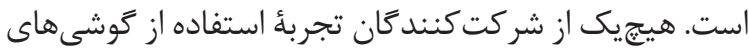

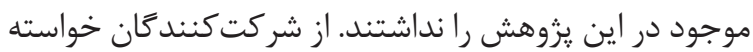

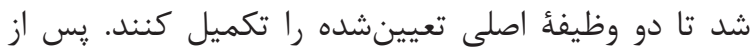

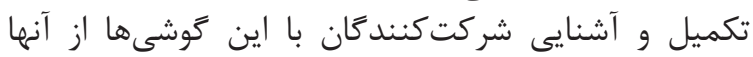

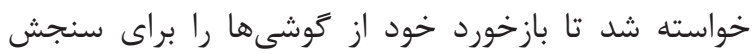

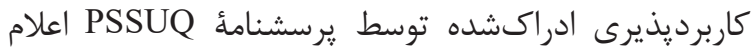

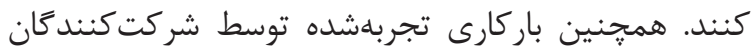

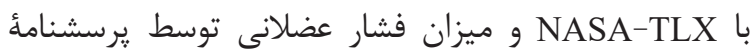
1 Borg CR

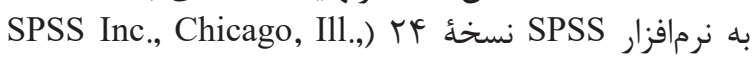

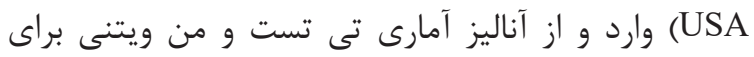
آناليز دادها استفاده شد. سطح معنى دارى در همأ آنا آزمونها

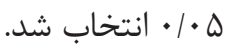

يافتهها

جدول امشخصات سن و تحصيلات كاربران سالمند راندان نشان مى دهد.

سنجش وظايف تعيينشده نشان مىدهد كه كه ميانكَين

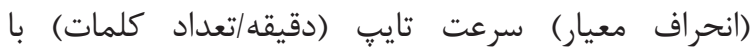

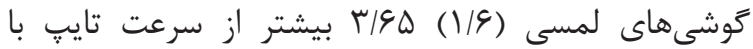


مقدار را نسبت به ساير اندامها نشان مىدهد (جدول سَ). با

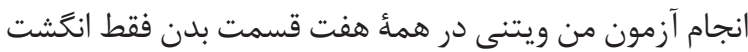

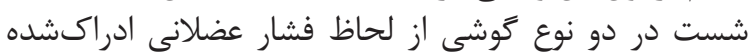

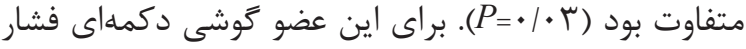

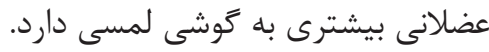

ميزان را بين ابعاد مختلف يرسشنامه NASA-TLX دارد

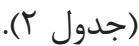

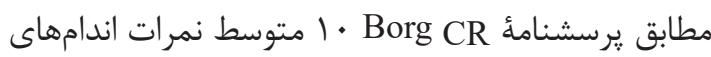

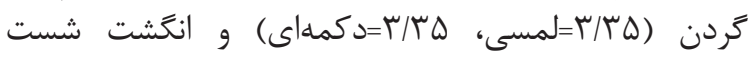

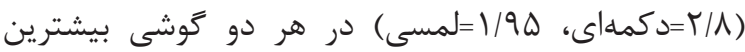

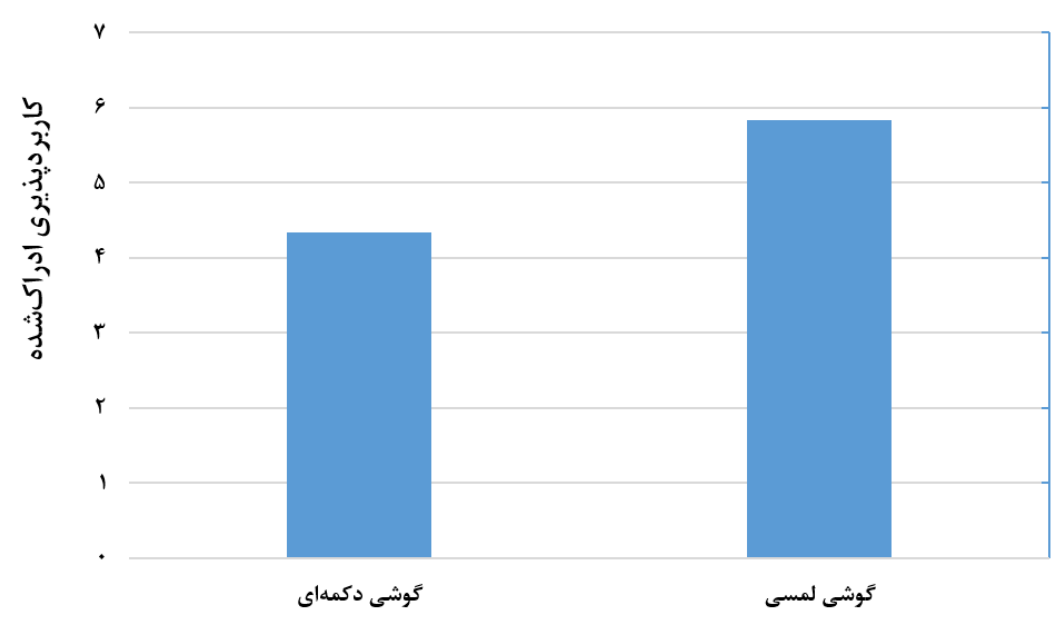

شكل r. متوسط كاربرديذيرى ادراكشده در دو گَشى لمسى و دكمهاى

جدول ا. مشخصات سن و تحصيلات كاربران سالمند

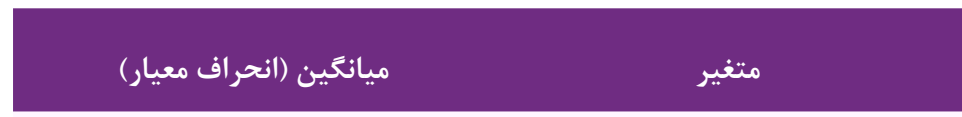

\begin{tabular}{|c|c|}
\hline$\varepsilon \Delta / r \Delta \pm(r / \varepsilon r)$ & سن (سال) \\
\hline$\%$ & تحصيلات (فوقدييلم و پِايينتر) \\
\hline
\end{tabular}

جدول r. ميانغين مقادير بهدست آمده از برسشنامهُ NASA-TLX

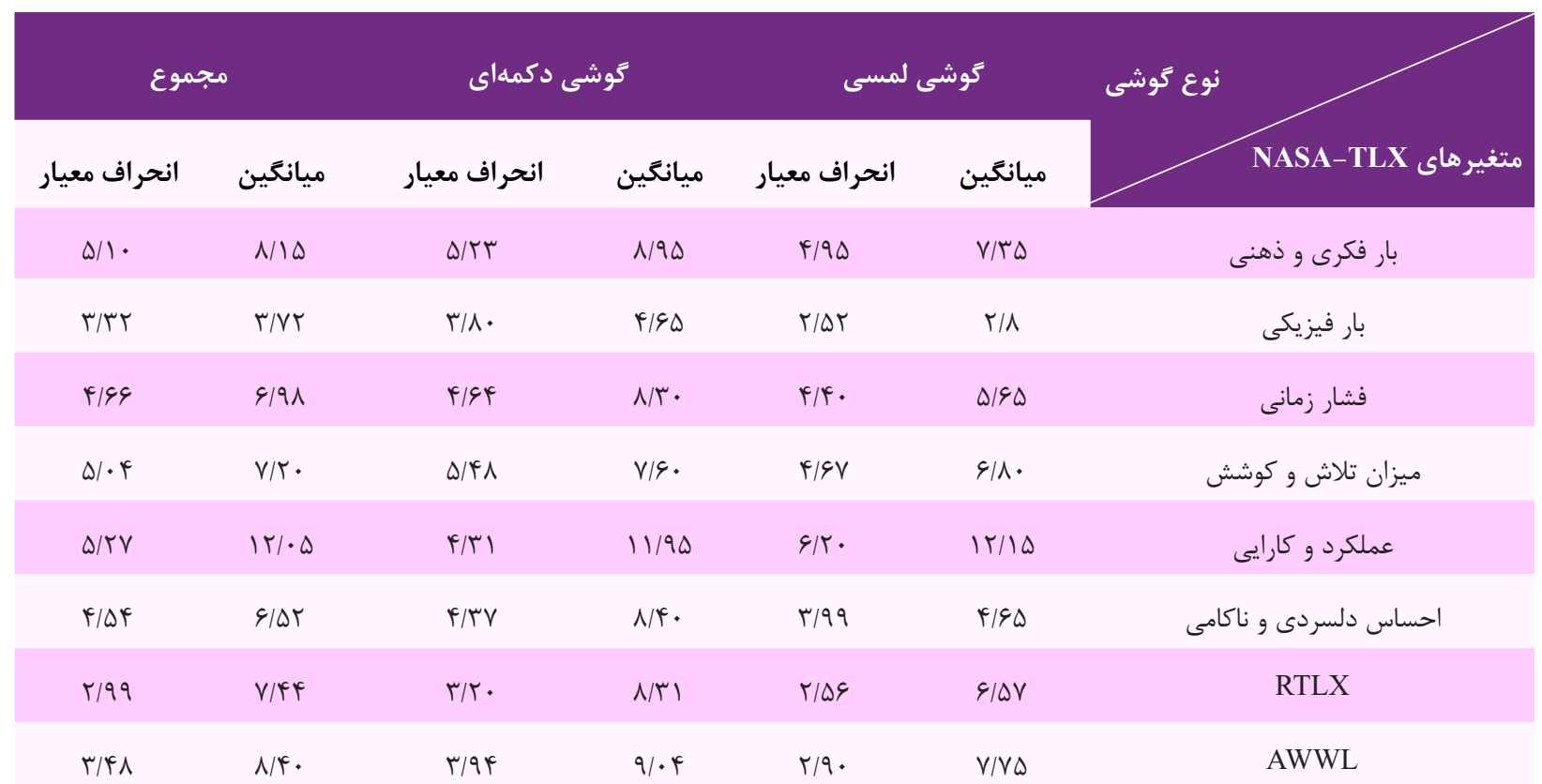


جدول r. ميانغين مقادير مقياس Borg CR 10 براى اندامهاى مختلف بدن

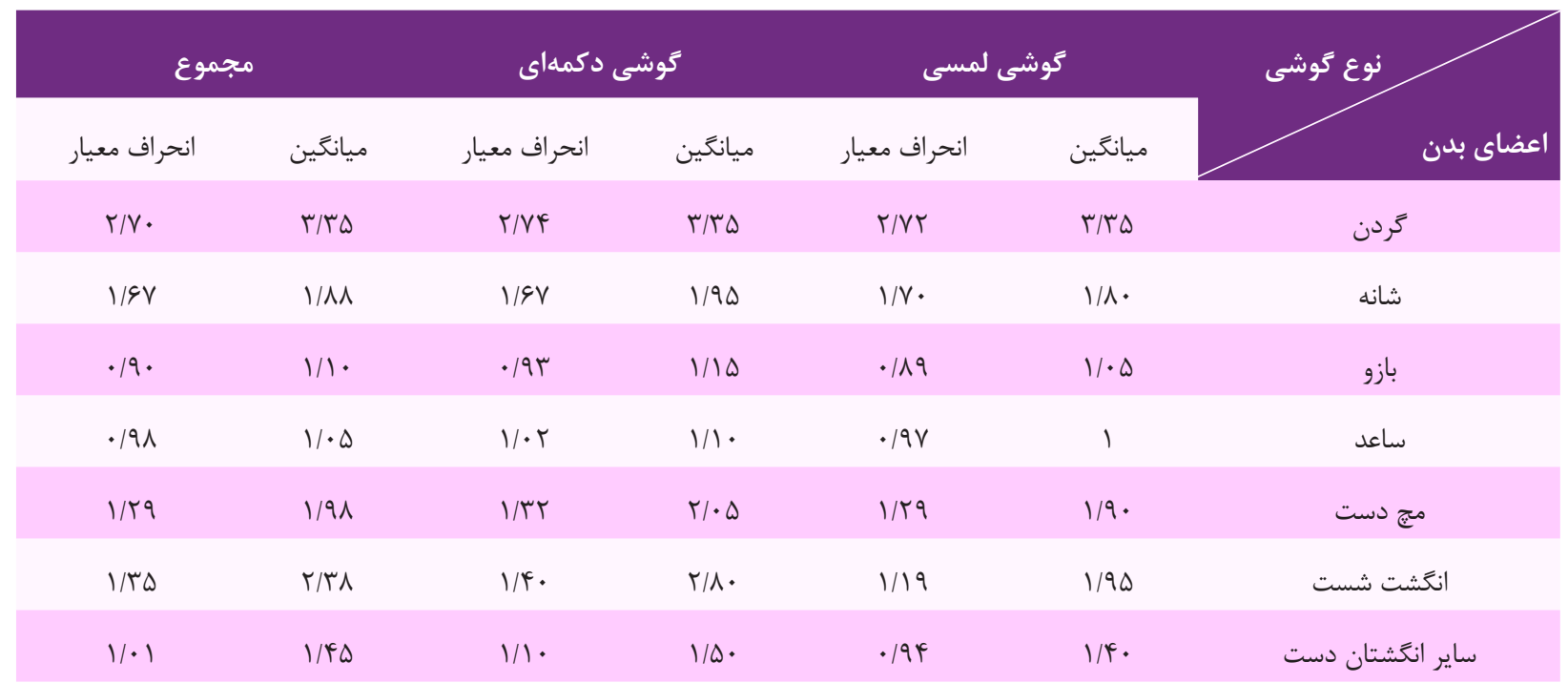

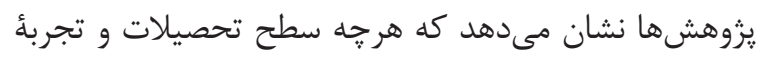

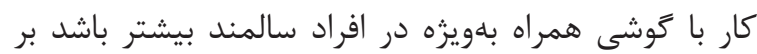

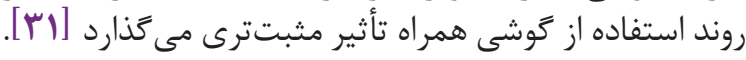

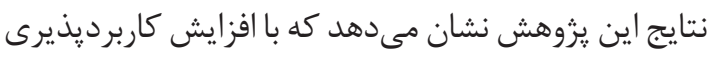

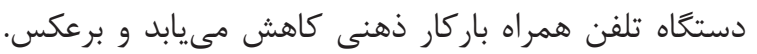

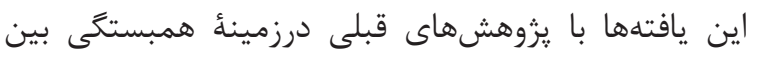

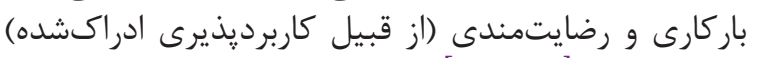

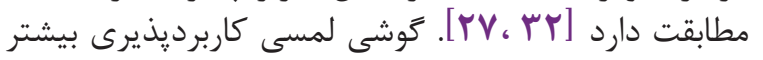

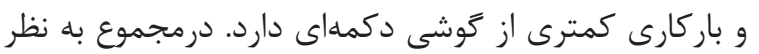

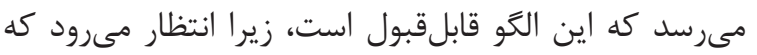

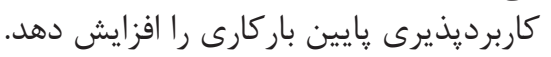

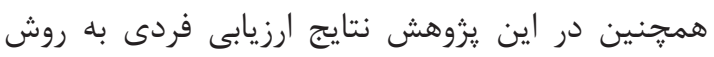

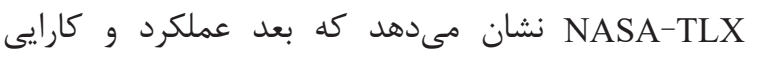

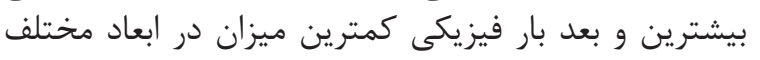

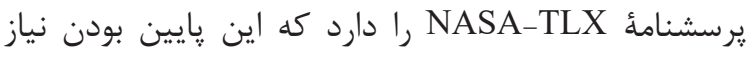

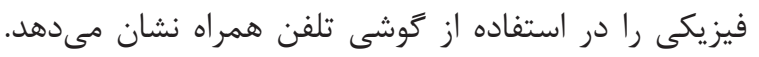

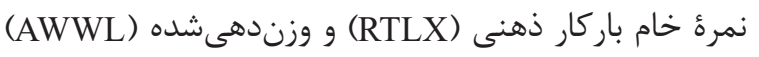

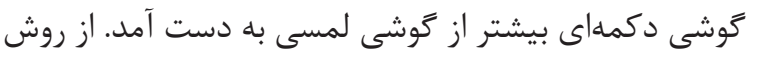

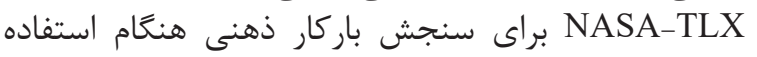

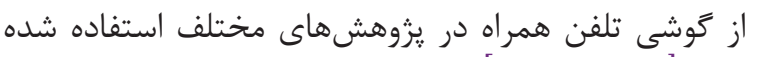

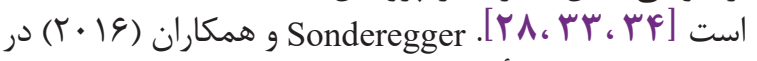

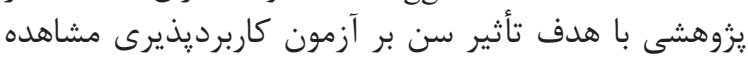

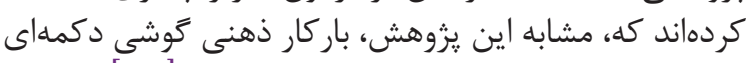

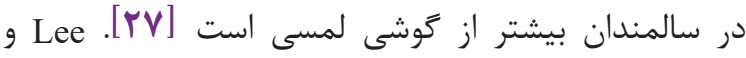

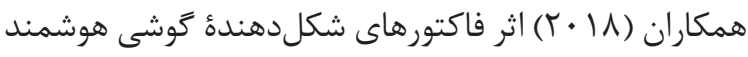

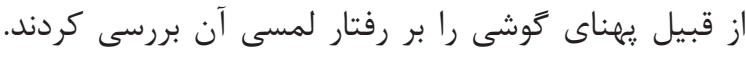

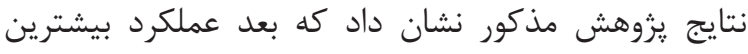

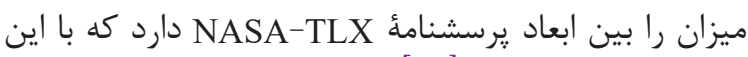

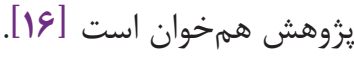

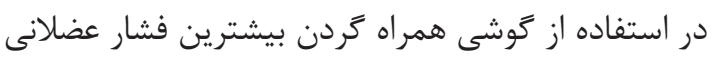

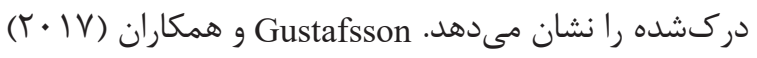

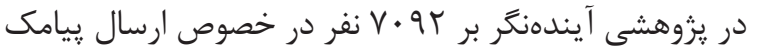

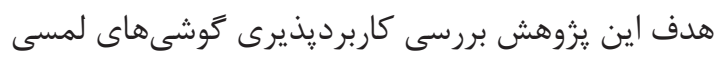

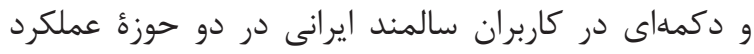

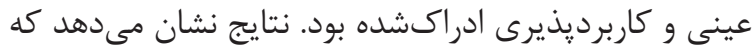

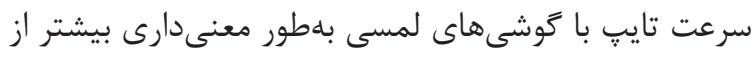

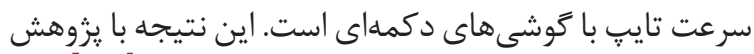
Sonderegger

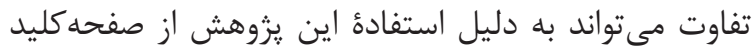

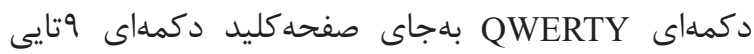

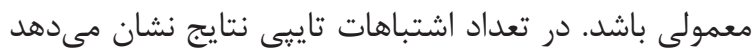

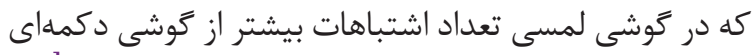

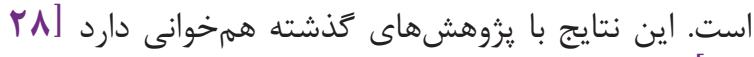
[IF،

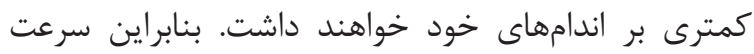

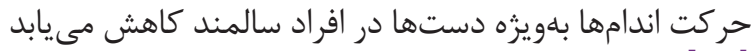

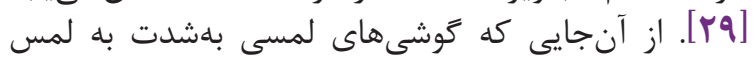

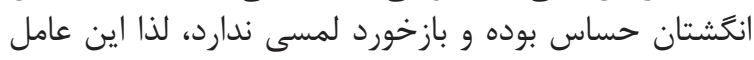

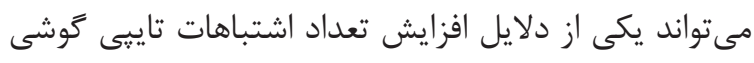

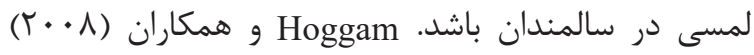

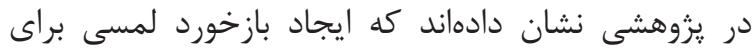

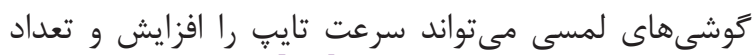

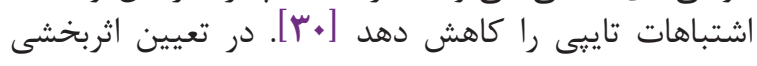

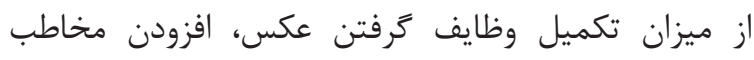

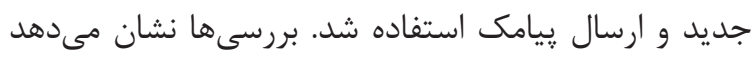

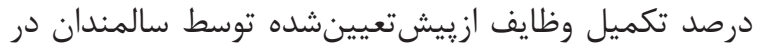

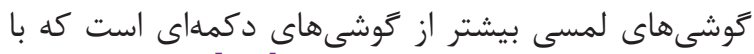

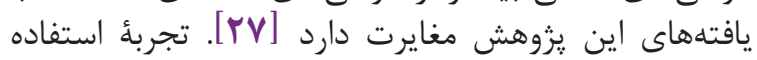

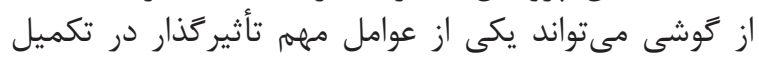

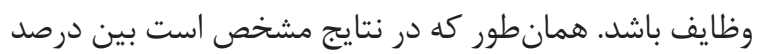

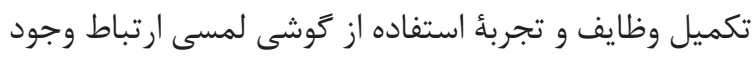

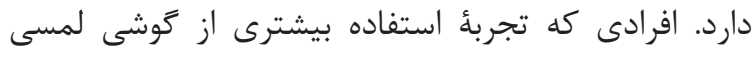

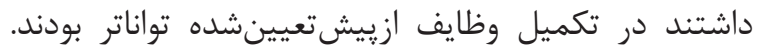


مىدهد كه براى جلوكيرى از اختلالات اسكلتى ـ عضلانى

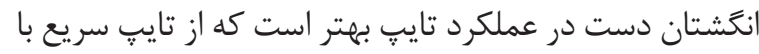

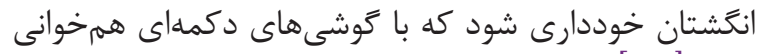

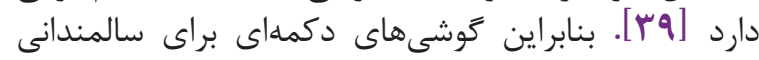

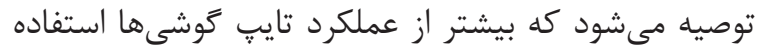
مى كنند.

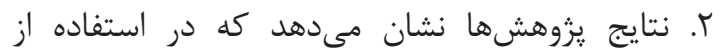

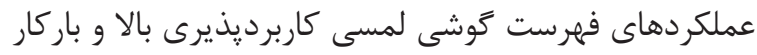

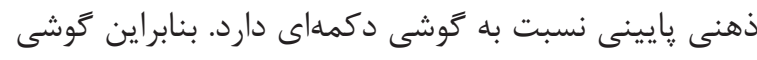

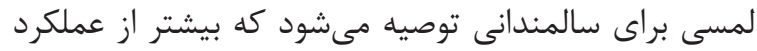

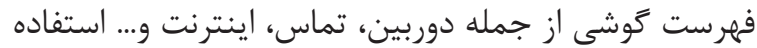

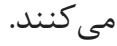

اين يزوهش روى جمعيت محدودى از سالمندان انجام

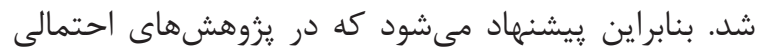

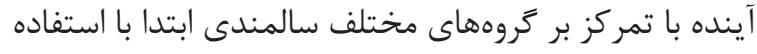

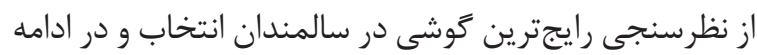

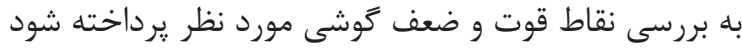

$$
\text { سياسگزارى }
$$

از همئ افر اد سالمند شركت كننده بر إى همكارى در اجراى اين طرح تقدير و تشكر مئشود.

$$
\text { تعارض در منافع }
$$

بين نويسندكان هيجَّونه تعارضى در منافع وجود ندارد.

\section{References}

1. World Health Organization. Global age-friendly cities: A guide. World Health Organization; 2007.

2. Rogers WA, Stronge AJ, Fisk AD. Technology and aging. Reviews of human factors and ergonomics. 2005 Jun;1(1):130-71.

3. Kroemer KH. 'Extra-Ordinary'Ergonomics: How to Accommodate Small and Big Persons, The Disabled and Elderly, Expectant Mothers, and Children. CRC Press; 2005 Aug 12.

4. Matthews G, Davies DR, Stammers RB, Westerman SJ. Human performance: Cognition, stress, and individual differences: Psychology Press; 2000.

5. Chua SL, Chen DT, Wong AF. Computer anxiety and its correlates: a meta-analysis. Computers in human behavior. 1999 Sep 1;15(5):609-23.

6. Rosen LD, Weil MM. Computer anxiety: A cross-cultural comparison of university students in ten countries. Computers in Human Behavior. 1995 Mar 1;11(1):45-64.

7. Taveira AD, Choi SD. Review study of computer input devices and older users. Intl. Journal of Human-Computer Interaction. 2009 Jun 8;25(5):455-74.
و اختلالات اسكلتى ـ عضلانى در اندام فوقانى و گردن درد

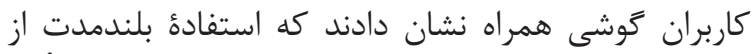

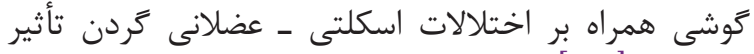

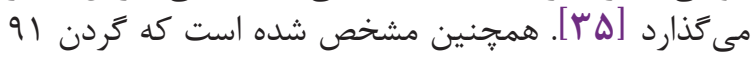

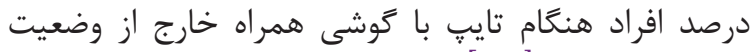

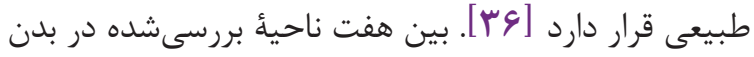

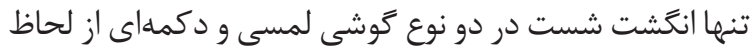

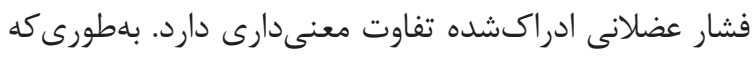

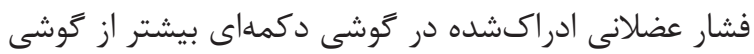

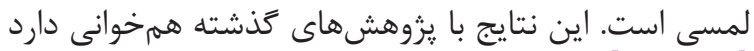

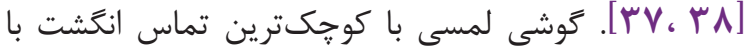

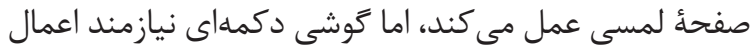

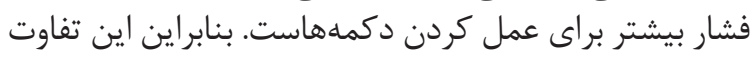
قابل فقبول است.

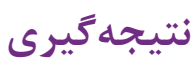

با توجه به گَسترش روزافزون كَشى همراه بين همأ

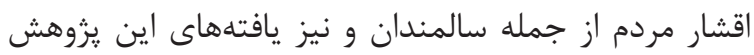

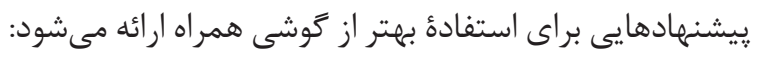

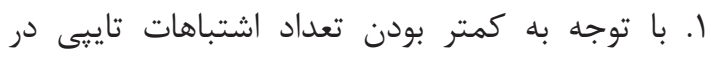

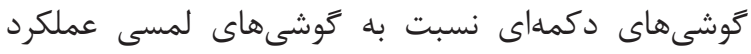

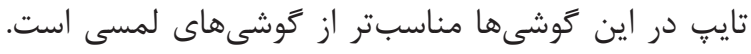

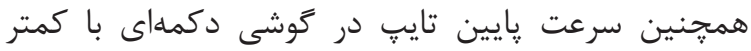

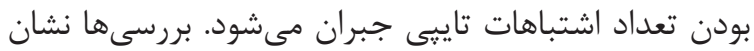

8. Social WA. Digital in 2018/Jan., Essential İnsight İnto İnternet, Social Media, Mobile And E-Commerce Use Around The World. Hootsuite.

9. Nielsen J. Usability 101: Introduction to usability. 2003

10. Safdari R, Shams Abadi AR, Pahlevany Nejad S. Improve Health of the Elderly People With M-Health and Technology. Iranian Journal of Ageing. 2018 Oct 15;13(3):288-99.

11. Nielsen J. Usability inspection methods. InConference companion on Human factors in computing systems 1994 Apr 28 (pp. 413-414). ACM.

12. Jordan PW. An introduction to usability. CRC Press; 1998 Jan 20.

13. Plaisant $\mathrm{C}$, Sears A. Touchscreen interfaces for alphanumeric data entry. InProceedings of the Human Factors Society Annual Meeting 1992 Oct (Vol. 36, No. 4, pp. 293-297). Sage CA: Los Angeles, CA: SAGE Publications.

14. Gustafsson E, Coenen P, Campbell A, Straker L. Texting with touchscreen and keypad phones-A comparison of thumb kinematics, upper limb muscle activity, exertion, discomfort, and performance. Applied ergonomics. 2018 Jul 1;70:2329. 
15. Chang J, Choi B, Tjolleng A, Jung K. Effects of button position on a soft keyboard: Muscle activity, touch time, and discomfort in two-thumb text entry. Applied ergonomics. 2017 Apr 1;60:28292.

16. Lee SC, Cha MC, Hwangbo H, Mo S, Ji YG. Smartphone form factors: Effects of width and bottom bezel on touch performance, workload, and physical demand. Applied ergonomics. 2018 Feb 1;67:142-50.

17. Colle HA, Hiszem KJ. Standing at a kiosk: Effects of key size and spacing on touch screen numeric keypad performance and user preference. Ergonomics. 2004 Oct 22;47(13):1406-23.

18. Schedlbauer M. Effects of key size and spacing on the completion time and accuracy of input tasks on soft keypads using trackball and touch input. InProceedings of the Human Factors and Ergonomics Society annual meeting 2007 Oct (Vol. 51, No. 5, pp. 429-433). Sage CA: Los Angeles, CA: SAGE Publications.

19. Cafebazaar. Winter season developers cafebazaar report. 2018. https://s.cafebazaar.ir/1/reports/ Quarterly-Market-Review-Q4-96.pdf

20. MacKenzie IS, Soukoreff RW. Text entry for mobile computing: Models and methods, theory and practice. Human-Computer Interaction. 2002 Sep 1;17(2-3):147-98.

21. Lewis JR. IBM computer usability satisfaction questionnaires: psychometric evaluation and instructions for use. International Journal of $\mathrm{Hu}-$ man-Computer Interaction. 1995 Jan 1;7(1):5778 .

22. Lawshe $\mathrm{CH}$. A quantitative approach to content validity 1. Personnel psychology. 1975 Dec;28(4):563-75.

23. Hart SG, Staveland LE. Development of NASA-TLX (Task Load Index): Results of empirical and theoretical research. InAdvances in psychology 1988 Jan 1 (Vol. 52, pp. 139-183). North-Holland.

24. Mohammadi M, Mazloumi A, Zeraati H. Designing questionnaire of assessing mental workload and determine its validity and reliability among ICUs nurses in one of the TUMS's hospitals. Journal of School of Public Health and Institute of Public Health Research. 2013 Nov 15;11(2):87-96.

25. Borg G. Psychophysical scaling with applications in physical work and the perception of exertion. Scand J Work Environ Health. 1990 Jan 1;16(Suppl 1):55-8.

26. Daneshmandi H, Choobineh A, Rajaee-Fard A. Validation of Borg's RPE 6-20 Scale in Male In- dustrial Workers of Shiraz City Based on Heart Rate. Jundishapur Scientific Medical Journal, . 2012; 11(1): 1-10.

27. Sonderegger A, Schmutz S, Sauer J. The influence of age in usability testing. Applied Ergonomics. 2016 Jan 1;52:291-300.

28. Crandall JM, Chaparro A. Driver distraction: Effects of text entry methods on driving performance. InProceedings of the human factors and ergonomics society annual meeting 2012 Sep (Vol. 56, No. 1, pp. 1693-1697). Sage CA: Los Angeles, CA: Sage

29. Carmeli E, Patish H, Coleman R. The aging hand. The Journals of Gerontology Series A: Biological Sciences and Medical Sciences. 2003 Feb 1;58(2):M146-52.

30. Hoggan E, Brewster SA, Johnston J. Investigating the effectiveness of tactile feedback for mobile touchscreens. InProceedings of the SIGCHI conference on Human factors in computing systems 2008 Apr 6 (pp. 1573-1582). ACM.

31. Ma Q, Chan AH, Chen K. Personal and other factors affecting acceptance of smartphone technology by older Chinese adults. Applied ergonomics. 2016 May 1;54:62-71.

32. Hornbæk K, Law EL. Meta-analysis of correlations among usability measures. InProceedings of the SIGCHI conference on Human factors in computing systems 2007 Apr 29 (pp. 617-626). ACM.

33. Chalil Madathil K, Koikkara R, Gramopadhye AK, Greenstein JS. An empirical study of the usability of consenting systems: iPad, Touchscreen and paper-based systems. InProceedings of the human factors and ergonomics society annual meeting 2011 Sep (Vol. 55, No. 1, pp. 813-817). Sage CA: Los Angeles, CA: SAGE Publications.

34. Dunlop M, Levine J. Multidimensional pareto optimization of touchscreen keyboards for speed, familiarity and improved spell checking. InProceedings of the SIGCHI Conference on Human Factors in Computing Systems 2012 May 5 (pp. 2669-2678). ACM.

35. Gustafsson E, Thomée S, Grimby-Ekman A, Hagberg M. Texting on mobile phones and musculoskeletal disorders in young adults: a fiveyear cohort study. Applied ergonomics. 2017 Jan 1;58:208-14.

36. Gold JE, Driban JB, Thomas N, Chakravarty T, Channell V, Komaroff EG. Postures, typing strategies, and gender differences in mobile device usage: An observational study. Applied ergonomics. 2012 Mar 1;43(2):408-12.

37. Berolo S, Wells RP, Amick III BC. Musculoskel- 
etal symptoms among mobile hand-held device users and their relationship to device use: a preliminary study in a Canadian university population. Applied Ergonomics. 2011 Jan 1;42(2):3718.

38. Kietrys DM, Gerg MJ, Dropkin J, Gold JE. Mobile input device type, texting style and screen size influence upper extremity and trapezius muscle activity, and cervical posture while texting. Applied ergonomics. 2015 Sep 1;50:98-104.

39. Gustafsson E. Ergonomic recommendations when texting on mobile phones. Work. 2012 Jan 1;41(Supplement 1):5705-6. 